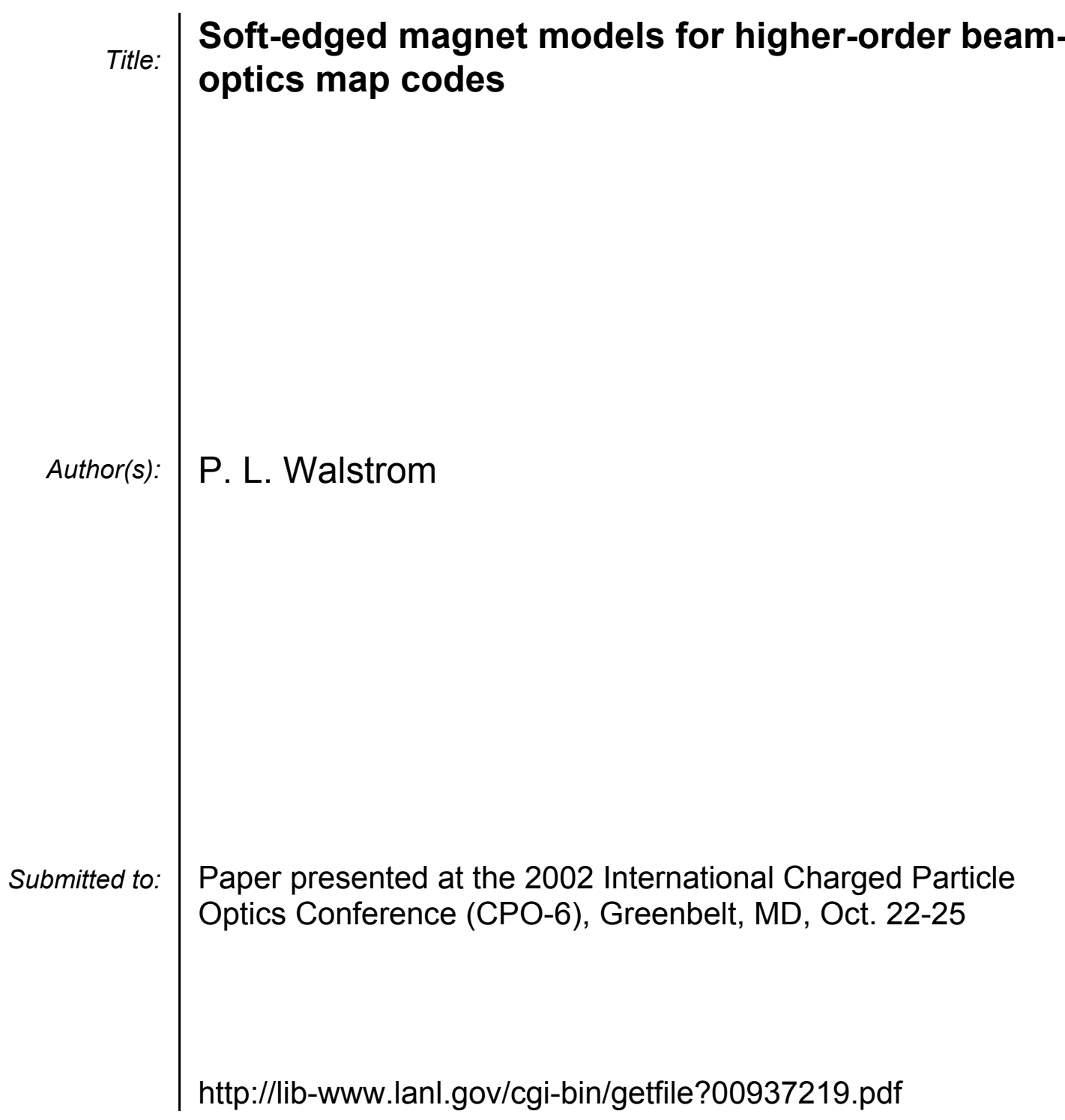
Laboratory requests that the publisher identify this article as work performed under the auspices of the U.S. Department of Energy. Los Alamos National Laboratory strongly supports academic freedom and a researcher's right to publish; as an institution, however, the Laboratory does not endorse the viewpoint of a publication or guarantee its technical correctness. 


\title{
Soft-edged magnet models for higher-order beam-optics map codes*
}

\author{
P. L. Walstrom \\ Los Alamos National Laboratory, Los Alamos, NM 87545 USA
}

Continuously varying surface and volume source-density distributions are used to model magnetic fields inside of cylindrical volumes. From these distributions, a package of subroutines computes on-axis generalized gradients and their derivatives at arbitrary points on the magnet axis for input to the numerical map-generating subroutines of the Lie-algebraic map code Marylie. In the present version of the package, the magnet menu includes: 1 . cylindrical current-sheet or radially thick current distributions with either open boundaries or with a surrounding cylindrical boundary with normal field lines (which models highpermeability iron), 2. Halbach-type permanent mutipole magnets, either as sheet magnets or as radially thick magnets, 3 . modeling of arbitrary fields inside a cylinder by use of a fictitious current sheet. The subroutines provide on-axis gradients and their $z$ derivatives to essentially arbitrary order, although in the present $3^{\text {rd }}$ and $5^{\text {th }}$ order Marylie only the $0^{\text {th }}$ through $6^{\text {th }}$ derivatives are needed. The formalism is especially useful in beam-optics applications, such as magnetic lenses, where realistic treatment of fringefield effects is needed.

\section{Introduction}

The term soft-edged is used here to refer to magnet models with realistic treatment of fringe fields that produce fully 3-D solutions of the Laplace equation. This approach is in contrast to that of the less exact but very useful hard-edged models, in which the central field is 2-D and abruptly drops to zero at the ends of the magnet. Transfer maps for hard-edged models can include higher-order effects to a limited degree. For example, $3^{\text {rd }}$-order aberrations can be added in a consistent way to transfer maps for hardedged quadrupoles, but $5^{\text {th }}$-order edge kicks cannot be defined for them.

The development of the soft-edged magnet models that are the subject of this paper dates back to the Neutral Particle Beam (NPB) program at Los Alamos. The NPB, which was part of the Strategic Defense Initiative of the late eighties, required higher-order optics computation for design of machines to produce low-divergence neutral particle beams. The beams in these devices were $\mathrm{H}^{-}$beams through the accelerator and final magnetic optics, and were neutralized at the very end of machine. The final optics consisted of the eyepiece, telescope, and steering magnet. Realistic softedged models were especially important for the large-bore telescope, which was a permanentmagnet quadrupole triplet, and steering magnet, which was a rapidly-slewing, relatively weakfield air-core single-layer winding.

* LA-UR-02-6780
The basic magnetic optics design tools used at LANL in the NPB were $3{ }^{\text {rd }}$-order Marylie [1], the $11^{\text {th }}$-order transfer-map code TLIE, and various effectively infinite-order particle tracking codes based on the soft edged field models used in the map codes. At the start of the program, $3^{\text {rd }}$-order Marylie already had a routine for soft-edged on-axis gradients of Halbach-type permanent magnet quadrupoles. This routine is used in conjunction with a routine for numerical integration of map coefficients to produce Marylie transfer maps for soft-edged magnets. This capability was used in initial design of the permanent-magnet telescope, including the design of octupoles for correction of $3^{\text {rd }}$ order aberrations. However, for analysis of $5^{\text {th }}$ and higher-order effects in the telescope, and for design and analysis of the steering magnet, for which no good field model existed, new capabilities were needed. A set of subroutines for computing soft-edged on-axis generalized gradients and their derivatives to high order in $z$, the beamline axial coordinate, for magnets of arbitrary multipole index $m$ were developed to meet the need. This subroutine package was used in a special $5^{\text {th }}$-order version of Marylie. As a byproduct of this work, a new approach to design of windings that produce 3-D fields of pure $\cos \mathrm{m} \phi$ symmetry was also developed. In the special LANL version of Marylie, the softedged routines are called by a routine (COILS) 
that provides a flexible framework for stacking magnets of arbitrary multipole index with overlapping fields [2].

The on-axis gradient subroutine package initially included current-sheet magnets with various winding patterns and thick and thin Halbach-type permanent multipole magnets. Later additions included radially thick windings and a model for a coaxial cylindrical boundary of infinite magnetic permeability. Finally, in order to be able to model arbitrary magnets (e.g., conventional iron-pole-piece magnets, etc.) a capability was added for computing on-axis gradients from effective sources derived from field data on a reference cylinder that are entered as a file. This extends the capability of the routines to essentially any magnet except strongly curved dipoles.

\section{Where soft-edged field models matter}

Reference has already been made to the NPB program, where high-order soft-edged field models were essential to the understanding of the beam optics. In general, soft edged magnet models are essential in the design and analysis of all high-quality quadrupole magnetic lens systems. Soft edged fields are needed even in the first-order (linear) optics of the problem, in order to get the drifts and magnet strengths right (one can always find hard-edge magnets and drifts that give the same optics as the soft-edged models, but only after the fringe-field profiles are known). The limitations of hard-edged models in the linear optics problem are even greater if the fields of the quadruples overlap. Moreover, the $3^{\text {rd }}$-order hard-edged aberrations given by hard-edged models are only approximate (although, in many cases good to $15 \%$ or so). The soft edged models are therefore needed to get the exact $3^{\text {rd }}$ order aberrations and required to compute $5^{\text {th }}$ and higher-order quadrupole aberrations.

Some other magnetic beam-optics systems for which soft-edged field models are needed are wigglers, interaction regions in high-energy colliders, and magnetic spectrometers.

\section{The on-axis generalized gradient}

From the point of view of numerical transfer-map computation in straight-axis systems, the on-axis generalized gradient $g_{m}(z)$ and its $z$ derivatives completely define the magnet. If the field of a magnet contains more than the fundamental Fourier component (for example, in a soft-edged combined function magnet or in modeling of error fields), each Fourier component (multipole index $m$ ) is treated as a separate magnet in the coil stack and has a separate gradient function $g_{m}(z)$. Also, if skew field components with a particular $m$ value appear in the problem, they are also treated as separate magnets with separate gradient functions. Gradient computation for skew components is the same as for normal components; the different angular dependence of their fields is taken into account in the map integration routines.

In defining the on-axis generalized gradient, we start with the magnetic scalar potential, which, for normal field components, is defined to be

$$
V_{m}(r, \phi, z)=\sum_{m=0}^{\infty} \sin m \phi V_{m}(r, z)
$$

For skew components, $\cos m \phi$ replaces $\sin m \phi$. We then define $g_{m}(z)$ to be

$g_{m}(z)=\lim _{r \rightarrow 0} \frac{m V_{m}(r, z)}{r^{m}}$

The factor of $m$ is included Eq. 2 to make the definition consistent with the usual definition of the quadrupole gradient, for which $m=2$.

If $g_{m}(z)$ is analytic everywhere except at isolated singularities, the scalar potential can be reconstructed everywhere in space (except at the singularities) by the following integral [3]:

$V_{m}(r, z)=\frac{(m-1) !(2 r)^{m}}{\pi(2 m-1) ! !} \int_{0}^{\pi} g_{m}(z+i r \cos t) \sin ^{2 m} t d t$

Equation 3 is useful in for studying, by either analytical or numerical means, the off-axis $(r, z)$ dependence of the potentials that arise from various choices of ad-hoc functions for the onaxis gradient, for example, the tanh function, which will be discussed later in this paper. It is the 3-D analog of analytic continuation of the midplane magnetic field in 2-D problems. For example, if the midplane magnetic field in a 2-D problem has only the component $B_{y}$, which is given by an analytic function $B_{y}=f(x)$, then the field everywhere is given by the expression $B_{y}+i B_{x}=f(x+i y)$.

Taylor expansion in $r$ of the integrand of Eq. 3 and explicit evaluation of the resulting elementary integrals in $t$ gives the well-known 
infinite-series expression for the scalar potential in terms of $g_{m}$ and its $z$ derivatives:

$$
V_{m}(r, z)=\sum_{n=0}^{\infty} \frac{(m-1) !}{(m+n) ! n !}\left(-\frac{r^{2}}{4} \frac{d^{2}}{d z^{2}}\right)^{n} g_{m}(z)
$$

Eq. 4 is the basis for order-by-order expansion of the contribution of magnetic vector potential and its derivatives to the total Hamiltonian used in numerical map generation. In a gauge with $\mathrm{A}_{\phi}=0$, the vector potential is

$$
\begin{aligned}
& A_{r}(r, z)=-\cos m \phi \frac{r}{m} \frac{\partial V_{m}(r, z)}{\partial z}, \\
& A_{z}(r, z)=\cos m \phi \frac{r}{m} \frac{\partial V_{m}(r, z)}{\partial r} .
\end{aligned}
$$

Equation 4 is also useful for field calculation in particle tracking, if the field evaluation point is inside the convergence volume of the series. The radius of convergence of Eq. 4 is $z$-dependent and is determined by the nearest singularity of $g_{m}$ in the complex plane. Elementary sources in the present Fourier angular decomposition of the field are sinusoidal ring sources: rings coaxial with the beamline axis with a line monopole or dipole density varying as $\sin m \phi$ or $\cos m \phi$. For a point at $(r, \zeta)$ and ring source at $(a, z)$, the radius of convergence of Eq. 4 is $\left[a^{2}+(\zeta-z)^{2}\right]^{1 / 2}$.

\section{Source-based field models}

Basic potential theory tells us that the magnetic field inside of a source-free volume due to real sources outside of it (current-carrying windings, iron, etc.) can be exactly replicated by a distribution of fictitious sources (magnetic monopoles and/or dipoles) on its surface. The distribution is in general a continuous one. However, even if the true sources are only approximated and are discontinuous, the field computed from the approximate sources will be curl and divergence free. Moreover, errors due to the discretization of the sources, etc. fall off rapidly as the field point moves away from the source surface (the method is stable to errors). The most stable approach is to use field data on a surface that encloses the field volume of interest for beam optics (i.e., where the beam goes) and use it to find sources on the same surface or on a surface inside of it that contains the beam. By contrast, fitting field data near the axis to trial functions and extrapolating the fit outward is unstable, even if the fit is analytic (curl and divergence free). In any case, one must be aware of the analytic behavior of the functions of $(r, z)$ used in fitting field data (know the locations of singularities) to ensure that the fields are valid over the beam volume.

\section{Shape functions and surface currents}

This subject is treated in more detail in Ref [4]. Currents on the surface of a cylinder of radius $a$ are represented by a radially pointing dipole density distribution $\psi(\phi, z)$ (double layer in classical potential theory). A single angular Fourier component of the density has the form

$\psi_{m}(\phi, z)=\sin m \phi f_{m}(z)$

The fields from these currents have only the $m$ th Fourier component. Winding layouts that produce 3-D fields with pure $\sin m \phi$ symmetry can be designed by these methods (turns are curves of constant $\psi_{m}(\phi, z)$. The components of current are

$j_{z}(\phi, z)=\cos m \phi \frac{m}{a} f_{m}(z)$

$j_{\phi}(\phi, z)=-\sin m \phi f_{m}^{\prime}(z)$

This is a special case of defining currents by $\mathbf{J}=\nabla \times \mathbf{M}$, which guarantees divergence-free currents and curl-free fields. If the shape function has a zone where it is constant in $z$, the current density component $j_{z}$ in the zone has the usual 2-D $\cos m \phi$ dependence, and $j_{\phi}=0$. An example of a winding that produces 3-D field with pure $\sin m \phi$ symmetry is shown in Fig. 1 . The shape function used to produce it is shown in Fig. 2.

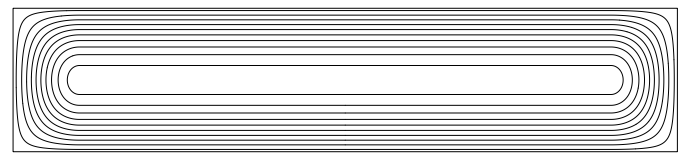

Fig. 1. One pole of a winding that produces a pure $\sin m \phi 3-D$ field (in the limit of an infinite number of turns).

In view of the above, the dipole-density distribution $\psi_{m}(\phi, z)$ can also be called the stream function for currents, using the terminology of 2-D potential flow theory. It can be decomposed 


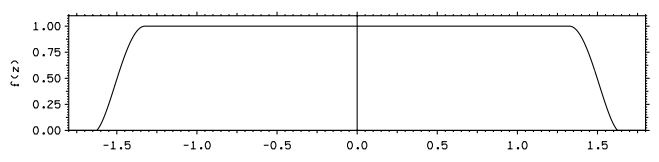

Fig. 2. The shape function used to produce the winding of Fig. 1 (see Eq. 9).

into elementary sinusoidal source rings of radius $a$ with weight $f_{m}(z)$. The scalar potential for one of these rings at $(a, z)$ is

$V_{m}(r, \phi, \zeta)=-\frac{a}{2 \pi} \frac{\partial}{\partial a} \frac{1}{\sqrt{a r}} Q_{m-1 / 2}\left(\frac{a^{2}+r^{2}+(\zeta-z)^{2}}{2 a r}\right)$

In Eq. $10, Q_{\mathrm{m}-1 / 2}$ is a Legendre function of the second kind. The potential is the 3-D analog of the 2-D line dipole potential. Near the axis, $V_{m}$ varies as $r^{m}$. The on-axis gradient for one of the dipole ring sources is obtained by taking the limit defined by Eq. 2 and is

$$
g_{m}(\zeta)=-\frac{(2 m-1) ! !}{(m-1) ! 2^{m+1}} a \frac{\partial}{\partial a} \frac{a^{m}}{\left[a^{2}+(\zeta-z)^{2}\right]^{m+1 / 2}}
$$

Similar expressions appear for Halbach PPM distributions (see Ref. 4). This expression and its $z$ derivatives are the basic building block for the LANL soft-edged gradient routines. When the shape function is represented by piecewisecontinuous polynomials, the integrals over $z$ of their products with the right-hand side of Eq. 11 and its derivatives can be evaluated in terms of simple algebraic expressions that can be summed to find the total gradient. When an arbitrary shape function is represented by a file of discrete $\left(z, f_{m}\right)$ pairs, a routine that generates a piecewise continuous quadratic interpolant to the data is used.

\section{Finding effective sources from field data: direct transforms vs. fitting methods}

Generally, we prefer direct transform approaches. They are easiest to implement in cases where the surface can be obtained setting one of the coordinates in which the Laplace equation is separable equal to a constant. Two examples of this are infinite cylinders and spheres. Some counterexamples are rectangular boxes, cylinders with closed ends, coaxial cylinders with the ends connected by an annular ring, etc.
Fitting methods can work well if done correctly, and are the only way to proceed if field data are limited. They are also useful if the field-data surface does not allow transform methods to be used. A good fitting approach is to enclose the field volume of interest with a surface, place sources with unknown strengths on the surface, and find source strengths by a least-squares fit to the field data on points on and inside the surface. One should have many more field points than source strengths, etc. This approach can work well if the field data are outside of a surface that surrounds the field volume of interest.

The only transform approach used to date in the LANL soft-edged models is Fourier transforms in $z$ for field data on an infinite cylinder. This requires field data on a cylinder that have been Fourier-analyzed in angle to get the separate angular components, usually $B_{r}^{m}(z)$. For the Fourier transform in $z$ of these components, data effectively from $z=-\infty$ to $z=$ $+\infty$ are needed (in practice, data out to $z$ values with negligible measured fields). Fourier transform methods are also used to find equivalent current sheets to represent the iron contribution when windings are surrounded by a coaxial cylindrical iron boundary. These methods are described in more detail in the following section.

An alternative to both the fitting and transform methods exists. If all three components of the field are known on a surface (or if the normal field and the scalar potential are known), then monopole and normally pointing dipole source distributions are given directly by the normal field and the scalar potential on the surface [5]. This method is especially useful for arbitrary surfaces for which finite-element codes can provide the data as part of the output.

\section{Fourier-transform methods}

Each $m$ th Fourier angle component is treated as a separate problem. In treatment of field data, ${ }_{B_{r}} m(z)$ is first Fourier transformed in $z$ :

$b_{m}(k)=\frac{1}{2 \pi} \int_{-\infty}^{+\infty} B_{r}^{m}(a, z) e^{-i k z} d z$

Two methods can now be used to use the stored $b(k)$ values to get gradients. The approach taken by Dragt and Venturini [7] in a special version of Marylie is to perform the following back Fourier integral computation by numerical means during 
map integration to get $g_{m}(z)$ for each new $z$ value:

$g_{m}(z)=\int_{-\infty}^{+\infty} \frac{k^{m-1}}{2^{m}(m-1) ! I_{m}^{\prime}(k a)} b_{m}(k) e^{i k z} d k$

The LANL approach, called the effective current-sheet shape function method, is to compute the shape function for effective currents by a numerical back transform and store it as a file of $\left(z, f_{m}\right)$ pairs. Then the algorithms for current sheet sources can be used to get gradients. The shape function for the equivalent windings is

$$
f_{m}(z)=\int_{-\infty}^{+\infty} \frac{1}{k^{2} I_{m}^{\prime}(k a) K_{m}^{\prime}(k a)} b_{m}(k) e^{i k z} d k
$$

\section{Modeling single-ended fringe fields in long magnets}

In using the above transform methods, it is not efficient or accurate to treat the whole field of a long magnet (e.g. $L / a>10$ ) as a fringe field. The remedy is to match the 2-D central field on the cylinder with the field from a known distribution and to treat only the difference field with Fourier transform methods. For example, if the fringefield region of the long magnet in question is on the right of a long central field region with field $B_{r}=B_{0} \sin m \phi$, and the $z$ origin is taken to be in the middle of the fringe-field region, the gradient function

$g_{m}(z)=g_{0}\left[\frac{1}{2}-\frac{1}{2} \tanh \left(\frac{\pi z}{2 a}\right)\right]$

with $g_{0}=B_{0} a^{-m}$ can be used to cancel out all of the field except the fringe field. The tanh field is calculated with either Eq. 3 or Eq. 4 (the latter if $a$ is not too close to the source radii). When integrating map coefficients, the tanh gradient and the Fourier-transformed difference-field gradient are treated as separate magnets in the coil stack.

\section{A proposed new Marylie soft-edged magnet menu}

The present collection of LANL Marylie softedged gradient model routines has grown over the years and the nomenclature is rather complicated. The package is not part of the present official $3^{\text {rd }}$-order Marylie release version.
However, the routines have proven to be indispensable in the analysis of many optics problems. The coil stacking method in the LANL version, which allows superposition of magnets with arbitrarily overlapping fields with various $m$ values, etc., is a useful and powerful tool.

The option of entering shape functions in the form of files, together with use of the standalone program that converts measured fields into shape functions for fictitious current sheet windings, is presently available to model arbitrary straight-axis magnets. However, for many potential users, it is inconvenient to use this option in everyday application of the program, since field data for arbitrary magnets are not readily available. For this reason, a menu of soft-edge models based on stored real field data (measured or computed by finite-element codes) for a large range of representative magnets of various types and sizes would be a useful addition to the soft edged gradient package. This option would be added to the presently available ones.

\section{References}

[1] Alex J. Dragt, et. al., "MARYLIE 3.0 User's Manual, A Program for Charged Particle Beam Transport Based on Lie Algebraic Methods", available from the Physics Department, University of Maryland, College Park, Maryland 20742-4111.

[2] Peter Walstrom, Filippo Neri, and Tom Mottershead, "High Order Optics of Multipole Magnets", Proc. 1990 Linear Accelerator Conference, LANL pub. No. LA-12004-C, March, 1991.

[3] E. T. Whittaker and G. N. Watson, A Course in Modern Analysis, $4^{\text {th }}$ Ed., Cambridge University Press, 1996, p. 400. The original reference is J. Dougall, Proc. Edinburgh Math. Soc. XXXIV. (1916), pp. 176-196.

[4] P. L. Walstrom, "Dipole-Sheet Multipole Magnets for Accelerators", IEEE Transactions on Magnetics, Vol. 30, No. 4, July 1994.

[5] A. Dragt, P. Roberts, T. Stasevitch, and P. Walstrom, "Computation of Charged-Particle Transfer Maps for General Fields and Geometries Using Electromagnetic Boundary Value Data", paper presented at the 2000 International Computational Physics Conference Darmstadt, Germany, Sept. 11-14, 2000.

[7] Dragt, A. and M. Venturini, “Accurate Computation of Transfer Maps from 
Paper presented at the 2002 International Charged Particle Optics Conference (CPO-6), Greenbelt, MD, Oct. 22-25

Magnetic Field Data", Nuclear Instruments and Methods, A427, p. 387 (1999). 